\title{
Quantifying bubbling emission (ebullition) of methane from a rice paddy using high-time- resolution concentration data obtained during a closed-chamber measurement
}

\section{Masako KAJIURA ( $\nabla$ kajico@affrc.go.jp )}

Institute for Agro-Environmental Sciences, NARO https://orcid.org/0000-0001-9329-8966

Takeshi TOKIDA ( $\nabla$ tokida@affrc.go.jp)

Institute for Agro-Environmental Sciences, NARO https://orcid.org/0000-0001-7245-2952

\section{Research Article}

Keywords: $\mathrm{CH} 4$ flux, Growth stage, Plant-mediated flux, Portable gas analyzer, Transport pathway

Posted Date: April 6th, 2021

DOl: https://doi.org/10.21203/rs.3.rs-396475/v1

License: (c) (1) This work is licensed under a Creative Commons Attribution 4.0 International License. Read Full License

Version of Record: A version of this preprint was published at Journal of Agricultural Meteorology on October 10th, 2021. See the published version at https://doi.org/10.2480/agrmet.D-21-00022. 


\section{Abstract}

Methane $\left(\mathrm{CH}_{4}\right)$ produced in rice-paddy soil is transported to the atmosphere either via the rice plants or by bubbling events (ebullition); however, little is known about the frequency and intensity of bubbling $\mathrm{CH}_{4}$ emissions and the factors that affect them. We developed a method to quantify ebullition using hightime-resolution $(\sim 1 \mathrm{~Hz}) \mathrm{CH}_{4}$ concentration data obtained by closed-chamber measurements. Field measurements were conducted in a Japanese rice paddy at different rice growth stages: panicle formation (PF), booting (BT), and heading (HD). A dataset of 132 chamber measurements was used to develop and evaluate the method. A scripting file written in $\mathrm{R}$ programing language was used to automatically determine $\mathrm{CH}_{4}$ emissions via the two pathways. Plant-mediated $\mathrm{CH}_{4}$ emission intensity was constant during chamber deployment and was reflected as a steady linear increase in chamber $\left[\mathrm{CH}_{4}\right]$

with time or as a constant baseline in a flux time series. We found that the plant-mediated emission could be determined as the peak with the lowest flux intensity in the flux frequency distribution even if bubbling events occurred during the chamber deployment. The field measurement results in combination with established data processing protocols showed that at PF, ebullition contributed only $4 \%$ of the total emission, whereas it accounted for $32 \%$ and $60 \%$ of the total emission at BT and HD, respectively. In contrast, the plant-mediated flux variation among growth stages was smaller. Both ebullition and plantmediated emissions correlated significantly with air temperature at $\mathrm{HD}$, but the magnitude of the dependency was much higher for ebullition than for rice-mediated emission. These results demonstrate that ebullition occurs more frequently than has previously been thought, and the different transport pathways show varying degrees of dependency on plant phenological and environmental factors, thus underscoring the need to separately determine $\mathrm{CH}_{4}$ emissions via each transport pathway.

\section{Introduction}

Rice paddies are a globally important source of atmospheric $\mathrm{CH}_{4}$ (Ciais et al., 2013), a potent greenhouse gas and historically the second largest contributor to global warming after $\mathrm{CO}_{2}$ (Myhre et al., 2013).

Methane produced in paddy soils enters the atmosphere either through the aerenchyma tissue of the rice plants (Nouchi et al., 1990; Wang et al., 1997) or by ebullition of $\mathrm{CH}_{4}$-containing gas bubbles (Komiya et al., 2020; Schütz et al., 1989; Wassmann et al., 1996). Entrapped gas bubbles (rather than dissolved $\mathrm{CH}_{4}$ in soil solution) in rice paddies have been shown to represent a major $\mathrm{CH}_{4}$ inventory component at later rice growth stages, even in soils regarded as water saturated (Tokida et al., 2013). Many studies have shown a very high $\mathrm{CH}_{4}$ mixing ratio in gas bubbles in rice-paddy soils (Byrnes et al., 1995; Uzaki et al., 1991; Watanabe et al., 1994). Molecular diffusion of dissolved $\mathrm{CH}_{4}$ across the water-atmosphere interface can also occur, but that contribution is usually marginal (Butterbach-Bahl et al., 1997; Schütz et al., 1989, Komiya et al., 2015) because $\mathrm{CH}_{4}$ is a sparsely soluble gas and its diffusion in water is four orders of magnitude smaller than that in the gas phase. In addition, methanotrophic bacteria may consume $80-90 \%$ of the $\mathrm{CH}_{4}$ diffusing through the soil-water interface before it reaches the atmosphere (Banker et al., 1995; Frenzel et al., 1992). 
Quantifying $\mathrm{CH}_{4}$ ebullition separately from the total $\mathrm{CH}_{4}$ emission remains a challenging issue.

Conventionally, the ebullition rate has been estimated by trapping evolving gas bubbles in an inverted water-filled funnel placed over the soil surface (Holzapfel-Pschorn et al., 1986) or by using a chamber method where, to exclude rice-mediated emission, no rice plants are included in the chamber (Cheng et al., 2006; Komiya et al., 2015). These techniques intrinsically assume spatial homogeneity of bubbling emissions; however, more ebullition might occur in proximity to rice plants because they provide carbon sources for $\mathrm{CH}_{4}$ production via rhizodeposition and degradation of plant tissues (Tokida et al., 2011). Thus, the currently accepted notion that rice-plant mediated transport is the dominant emission pathway is open to question, especially when the rice plants are still small (Schütz et al., 1989; Wassmann et al., 1996), or at later growth stages, when root system decay may reduce the ability of rice to transfer $\mathrm{CH}_{4}$ (Tokida et al., 2013).

It is obvious that even when ebullition is to be measured, it is preferable to include rice plants in the measurement chamber, but with conventional gas sampling and analysis protocols, it is not possible to separate ebullition from plant-mediated transport of $\mathrm{CH}_{4}$. Recently, spectroscopic gas analyzers have been developed that can measure the $\mathrm{CH}_{4}$ concentration ([CH4]) with high precision and high time resolution $(\sim 1 \mathrm{~Hz})$ (e.g. Gogo et al., 2011). Tokida (2021) has developed a modified closed chamber method for measuring rice-paddy $\mathrm{CH}_{4}$ emission that uses a commercially available portable spectroscopic gas analyzer. With this method, ebullition is identified by a "jump" in the high-timeresolution record of $\left[\mathrm{CH}_{4}\right]$ in the chamber air. Motivated by these advancements in measurement techniques, in this study we aimed to develop systematic data handling and analytical procedures to distinguish $\mathrm{CH}_{4}$ ebullition from total emissions. We also investigated two factors potentially affecting ebullition: i) plant growth stage, and ii) temperature, i.e., an environmental factor well known to affect $\mathrm{CH}_{4}$ emission.

\section{Methods}

\subsection{Experimental field and rice cultivation}

We used an experimental paddy field located at the National Agriculture and Food Research Organization in Japan ( $\left.36^{\circ} 01^{\prime} 28^{\prime \prime} \mathrm{N}, 140^{\circ} 06^{\prime} 30^{\prime \prime} \mathrm{E}\right)$. We sowed pregerminated seeds of rice (Oryza sativa L., cv. Koshihikari) on April 15, 2020, in seedling trays, and raised the seedlings in an open field. Koshihikari seedlings (5-leaf age) were transplanted at a spacing of $15 \mathrm{~cm} \times 30 \mathrm{~cm}$ with three seedlings per hill on May 14. We prepared a total of 44 measurement plots ( 11 plots $\times$ four replicates) within a single field (10 $\mathrm{m} \times 50 \mathrm{~m})$, where each plot was an independent canopy consisting of 24 hills of rice plants $(1.2 \mathrm{~m} \times 0.9$ $\mathrm{m}$ ). For each replicate, 11 plots were placed every $5 \mathrm{~m}$ (center to center) along the longer side of the field, and the four replicates were placed every $2.3 \mathrm{~m}$ across the shorter dimension of the field. The field was continuously flooded from transplanting until the middle of the grain-filling stage. Heading initiated on July 26. In June, July, and August 2020, the monthly average air temperature was $22.4,23.5$, and $27.8^{\circ} \mathrm{C}$, 
respectively, and monthly precipitation was 199,229 , and $59 \mathrm{~mm}$, respectively. Air temperature ( $2 \mathrm{~m}$ above the ground level) was observed at a meteorological tower $200 \mathrm{~m}$ away from the experimental field.

\subsection{Measurement of $\mathrm{CH}_{4}$ emission}

Methane emission was measured successively in each plot by a closed-chamber method (Minamikawa et al., 2015) but with a substantial modification of the $\left[\mathrm{CH}_{4}\right]$ measurement procedure. $\left[\mathrm{CH}_{4}\right]$ was monitored on site at high time resolution (at intervals of $0.9 \mathrm{~s}$ ) by using a mobile gas analyzer (G4301, Picarro Inc., Santa Clara, CA, USA) instead of a gas chromatograph in the laboratory (Tokida, 2021). The chamber and analyzer were connected by two PTFE tubes; a chamber air sample was withdrawn from the chamber via one tube, and then, after it passed through the gas analyzer, it was re-injected into the chamber via the other tube. We used two chambers and adopted an alternating chamber strategy to make the most efficient use of the gas analyzer (Tokida 2021). A closed-top acrylic chamber (basal dimensions, $30 \mathrm{~cm} \times$ $60 \mathrm{~cm}$ ) was used to enclose four hills of rice plants at the center of each plot. Measurements were made over four days ( 1 replicate per day) at each of three growth stages: panicle formation (PF, leaf number index 84-87; June 29, 30, July 2, 3 for Reps 1, 2, 3, 4, respectively), booting (BT, flag leaf just fully expanded; July 21, 22, 24, 25), and full heading (HD; August 4, 5, 7, 8). Thus, a total of 132 measurements (11 plots $\times 4$ replicates $\times 3$ stages) were obtained during this study. At the PF stage, only a $60-\mathrm{cm}$-high chamber equipped with a fan was used, whereas at the BT stage, a double-deck chamber, consisting of a 20-cm-high lower chamber without a fan and a 60-cm-high upper chamber equipped with a fan was used. At the HD stage, the lower chamber was replaced with a 60-cm-high chamber equipped with a fan. Actual duration of the chamber closure differed from one measurement to another (1.6-34 minutes). We kept the chamber closed until we obtained a steady $\left[\mathrm{CH}_{4}\right]$ increase, not interrupted by the bubbling event, for at least 1 minute. Therefore, the chamber deployment time was basically longer as the bubbling event occurred more frequently (section 2.3).

\subsection{Separate quantification of $\mathrm{CH}_{4}$ emissions via rice plants and ebullition}

We estimated plant-mediated emission and ebullition separately by analyzing the high-time-resolution data of chamber $\left[\mathrm{CH}_{4}\right]$ (Fig. 1). Two patterns were observed in each $\left[\mathrm{CH}_{4}\right]$ time series: a steady linear increase, and sudden rapid increases (Fig. 2a, d). We assumed that the steady linear increase corresponded to the emission via rice plants, which occurs at a constant rate, and that the stepwise increases occurring over a short time were due to the release of bubbles, that is, ebullition (indicated by arrows in Fig. 2a, d). Ebullition was observed frequently, especially at the HD stage, and the intensity of 
ebullition could vary substantially from one bubbling event to another, even during a single chamber deployment. The total $\mathrm{CH}_{4}$ flux was calculated by the traditional regression analysis approach (Minamikawa et al., 2015), whereas the rate of plant-mediated emission was determined by a series of data processing steps, starting with noise reduction (see section 2.3.2) and followed by analysis of the flux frequency distribution (section 2.3.3). Finally, the bubbling emission (ebullition) was calculated as the difference between the two (section 2.3.4). Each data processing step is explained in detail below.

\subsubsection{Cleaning raw $\left[\mathrm{CH}_{4}\right]$ data}

Because the gas analyzer was operated continuously, the raw time-series data contained $\left[\mathrm{CH}_{4}\right]$ measurements in ambient air introduced in between the measurements from different plots. Therefore, we removed the data from unstable periods (gray bands in Fig. 2a, d) by analyzing the raw $\left[\mathrm{CH}_{4}\right]$ and smoothed flux data (obtained by differentiation of $\left[\mathrm{CH}_{4}\right]$ with time and applying an adequate smoothing procedure (i.e., by applying the 10-point moving average five times) to obtain "processed" [CH4] data (Fig. 1). The cleaning procedure is described in detail in the Supplementary Information.

\subsubsection{Moving-average protocols for noise reduction}

The plant-mediated emission, which is represented by a linear increase in $\left[\mathrm{CH}_{4}\right]$ (Fig. 2a, d), can be identified as the "flat background" when expressed as a time series of $\mathrm{d}\left[\mathrm{CH}_{4}\right] / \mathrm{dt}$ or in units of flux (Gogo et al., 2011). Therefore, we differentiated $\left[\mathrm{CH}_{4}\right]$ measured at $0.9 \mathrm{~s}$ intervals with respect to time and carried out the calculations necessary to transform the results into $\mathrm{CH}_{4}$ flux $\left(\mathrm{mg}-\mathrm{C} \mathrm{m}^{-2} \mathrm{~h}^{-1}\right)$ data using the air volume and temperature in the chamber (Minamikawa et al., 2015) (Fig. 1: "Convert to flux" on the right under "Processed [CH4] data").

However, the time-series flux data (at 0.9 s intervals) showed frequent fluctuations (Fig. 2b, e, light gray lines), indicating that the high-frequency measurements included substantial errors due to incomplete mixing of chamber air and to the analytical error of the gas analyzer. Therefore, to correctly determine the plant-mediated flux, we needed to reduce the noise (by smoothing) (Fig. 1: "Moving average" under "Convert to flux").

To smooth the data, we calculated moving averages. To identify the most appropriate protocol, we compared moving-average results obtained by applying different numbers of data points $(5,10,20$, or 30 points) and repetitions (1-9). As a result, we obtained 36 different smoothed flux datasets for each chamber measurement. Then, we computed the frequency distribution of each flux dataset by estimating the kernel density distribution and using it to determine the plant-mediated flux for that dataset (Fig. 2c, f) 
(see section 2.3.3). We then investigated the appropriateness of each moving-average protocol by examining the robustness of the plant-mediated flux (Fig. 2b, e, blue baseline, and Fig. 2c, f, solid red vertical line) estimated by using each combination (number of data points in the averaging window and number of applications of the moving average; see section 3.1).

\subsubsection{Plant-mediated emission: estimation from the kernel density distribution}

To determine the intensity of the plant-mediated flux (blue baseline in Fig. 2b, e), we converted each flux time series into the flux frequency distribution by estimating the kernel density distribution (Fig. 2c, f). We hypothesized that the baseline emission could be determined from the peak with lowest flux intensity in the frequency distribution (solid red lines in Fig. 2c, f). We then evaluated whether the estimated flux agreed with the baseline in the flux time series by visual inspection (blue lines in Fig. 2b, e).

In the kernel density estimation, the bandwidth (smoothing parameter) was set to 0.30 for all plots; this value was the median bandwidth selected for all 132 flux measurements (11 plots $\times 4$ replicates $\times 3$ stages) by the bw.nrd function in R ver 4.0.3 (R Core Team, 2020). We then identified local maxima (i.e., peaks) in the kernel density distribution by using the peaks function (with default parameters) in the splus2R package in $\mathrm{R}$ (Constantine and Hesterberg, 2021).

When little ebullition occurred, the plant-mediated flux could be identified as the sharp peak with the highest density in the kernel density distribution (solid red vertical line in Fig. 2c). When considerable bubbling emission occurred, the kernel density distribution showed multiple peaks, but the plant-mediated flux could be still determined as the local maximum (peak) with the lowest flux intensity (solid red vertical line in Fig. 2f).

\subsubsection{Total emission and ebullition}

The total emission was determined by the traditional regression approach; we estimated the rate of increase of $\left[\mathrm{CH}_{4}\right]$ (processed $\left[\mathrm{CH}_{4}\right]$ ) by a linear regression analysis (dashed lines in Fig. 2a, d) and converted it to the units of flux (mg- $\mathrm{C} \mathrm{h}^{-1} \mathrm{~m}^{-2}$ ) using the air volume and temperature in the chamber (Minamikawa et al., 2015). The rate of ebullition averaged over each chamber deployment was calculated as the difference between the total and plant-mediated emission (total emission minus plant-mediated emission). Note that we only estimated the intensity of bubbling averaged over each chamber deployment period, not that of each bubbling event. 


\subsection{Statistical analysis}

We investigated the relationship between $\mathrm{CH}_{4}$ fluxes via rice plants and via ebullition at each growth stage and air temperature by a simple correlation analysis using the Im function in R ver 4.0.3.

\section{Results And Discussion}

\subsection{Selection of the moving-average protocol and estimation of plant-mediated $\mathrm{CH}_{4}$ emission}

Comparison of the results obtained by using different smoothing protocols led us to conclude that application of a 10-point moving average five times was the most appropriate protocol for our dataset. The most stable estimates were obtained with a 10-point window; standard deviations of estimates obtained by applying moving averages with different window sizes one to nine times increased in the order 10, 20, 5, and 30 data points (the median standard deviation for the 132 flux measurements was $0.05,0.09,0.29$, and $0.35 \mathrm{mg} \mathrm{CH}_{4} \mathrm{~m}^{-2} \mathrm{~h}^{-1}$, respectively).

The estimated plant-mediated flux generally increased with the number of times the moving average was applied, but with a 10-point window, the estimated values reached a plateau with two applications for most plots and with five applications for all plots except one (Fig. 3, 10-point results). In contrast, adopting other averaging windows caused the values to increase continuously with the number of applications, especially at the HD stage (Fig. 3). Thus, with a 5-point moving average, the noise might not be sufficiently removed, whereas using a 20- or 30-point moving average might obscure fluxes resulting from bubbling events. Visual inspection confirmed that the fluxes estimated by using a 10-point moving average five times agreed well with the baselines of the flux time series (blue lines in Fig. 2b, e; see Fig. S2 for the results for all plots), indicating the validity of this protocol.

Note that the protocol for the moving average reported here may be specific to the current dataset. The best smoothing protocol may depend on many factors, including the analytical error of the gas analyzer, the chamber design, the efficiency of chamber air mixing by the fan, and the chamber deployment time. Therefore, investigators need to identify the best settings for themselves. Here, we argue only that with an appropriate window size and number of repeated applications of the moving average, the plant-mediated flux can be accurately determined.

\subsection{Temporal changes in the $\mathrm{CH}_{4}$ concentration during measurement}


The pattern of the $\left[\mathrm{CH}_{4}\right]$ increase in the measurement chamber changed as the rice plants grew (Fig. S1). The $\left[\mathrm{CH}_{4}\right]$ values increased linearly at the PF stage, whereas sudden rapid increases, superimposed on the steady linear increase, were observed in several plots at the BT stage and in most plots at the HD stage, indicating more frequent ebullition events.

\subsection{Contribution of ebullition to total emissions}

The field measurement results in combination with the established protocols for noise reduction (applying a moving average) and flux determination showed that total $\mathrm{CH}_{4}$ emissions were almost the same between the PF and BT stages but increased drastically from the BT to the HD stage (Fig. 4). Little ebullition occurred at the PF stage, accounting for only $4 \%$ of the total emission, but ebullition increased as the rice plants developed and accounted for $32 \%$ and $60 \%$ of the total emissions at the BT and HD stages, respectively. In contrast, the plant-mediated flux marginally differed among the growth stages investigated. A rough estimation of the seasonal $\mathrm{CH}_{4}$ emissions indicated that bubbling emission contributed $26 \%$ of the total emission during the growing season (see Supplementary information 3 , Table S1). This value is a conservative estimate because we assumed that no bubbling emission occurred at night, but in fact, bubbling emission can occur at night, at least to some extent (Komiya et al., 2020). Therefore, ebullition contributed substantially to the total seasonal emissions and occurred more frequently than has previously been inferred (Cicerone and Shetter 1981; Nouchi et al., 1990; ButterbachBahl et al., 1997).

\subsection{Effect of temperature on plant-mediated emission and ebullition}

Both plant-mediated and bubbling emissions correlated significantly with air temperature at the HD stage $(P<0.001$ for both) but no correlation was observed at the PF and BT stages (Fig. 5). These observations are consistent with previous studies of $\mathrm{CH}_{4}$ emissions from rice paddies in the same region of Japan, which reported the occurrence of diel variation in $\mathrm{CH}_{4}$ emissions only during the late reproductive stage (Minamikawa et al., 2012; Tokida et al., 2014). The positive correlation observed at the HD stage may be due to both the accumulation of trapped gas bubbles in the soil and an increase in the gas volume associated with increased air temperatures. The higher the temperature, the lower the water-solubility of $\mathrm{CH}_{4}$ would be, leading to an increase in the gas phase in the soil. Simultaneously, the gas phase would expand in accordance with Charles's law, leading to the release of bubbles that, because of buoyancy, cannot be retained in the soil matrix (Tokida et al., 2009). 
An increase in gas-phase volume may also enhance plant-mediated emission. The greater the bubble volume, the larger the root surface area in direct contact with bubbles would be, leading to an enhanced diffusive uptake of $\mathrm{CH}_{4}$ via the root and aerenchyma tissues of rice (Tokida et al., 2013). This possible mechanism might be reflected in the weak but significant correlation between plant-mediated emission and temperature at the HD stage $\left(r^{2}=0.26, P<0.001\right)$, but the temperature dependency of plant-mediated fluxes was much lower than that of bubbling emission, as shown by the smaller slope of the former (0.33 vs 1.63) in the correlation plot (Fig. 5).

\section{Conclusion}

We developed a method to separately quantify $\mathrm{CH}_{4}$ emissions via rice plants and ebullition in a high-timeresolution dataset of $\left[\mathrm{CH}_{4}\right]$ obtained by a closed-chamber method. Plant-mediated emission showed a small growth-stage dependency, whereas bubbling emissions drastically increased from the BT to the HD stage. The temperature dependency of the $\mathrm{CH}_{4}$ flux was clear for both pathways at the HD stage, but the temperature sensitivity of bubbling emissions was much higher. These different responses between the two pathways clearly indicate the need to determine pathway-dependent emissions for accurate observation and modeling of rice-paddy $\mathrm{CH}_{4}$ emissions.

\section{Acknowledgements}

We thank Takeru Saito of Ibaraki University, Japan, Xuping Ma of the Institute for Agro-Environmental Sciences, NARO, Japan, for their support during the field measurements. This study was supported by JSPS KAKENHI Grant Numbers JP16H06204, JP19K22921, JP19H03096, JP20J40189, and also based on results obtained from a project, JPNP18016, commissioned by the New Energy and Industrial Technology Development Organization (NEDO). The authors declare no conflicts of interest associated with this manuscript.

\section{References}

Banker BC, Kludze HK, Alford DP et al., 1995: Methane sources and sinks in paddy rice soils: Relationship to emissions. Agriculture, Ecosystems \& Environment, 53, 243-251. doi: 10.1016/0167-8809(94)00578-3

Butterbach-Bahl K, Papen H and Rennenberg H, 1997: Impact of gas transport through rice cultivars on methane emission from rice paddy fields. Plant, Cell \& Environment, 20, 1175-1183. doi: 10.1046/j.13653040.1997.d01-142.x

Byrnes BH, Austin ER and Tays BK, 1995: Methane emissions from flooded rice soils and plants under controlled conditions. Soil Biology \& Biochemistry, 27, 331-339. doi: 10.1016/0038-0717(94)00187-6 
Cheng W, Yagi K, Sakai $\mathrm{H}$ et al., 2006: Effects of elevated atmospheric $\mathrm{CO}_{2}$ concentrations on $\mathrm{CH}_{4}$ and $\mathrm{N}_{2} \mathrm{O}$ emission from rice soil: An experiment in controlled-environment chambers. Biogeochemistry, 77, 351-373. doi: 10.1007/s10533-005-1534-2

Ciais P, Sabine C, Bala G et al., 2013: Carbon and other biogeochemical cycles. In: Climate change 2013: The physical science basis. Contribution of working group I to the fifth assessment report of the intergovernmental panel on climate change. (ed by Stocker, TF, Qin D, Plattner G-K et al.). Cambridge University Press, Cambridge, United Kingdom and New York, NY, USA.

Cicerone RJ and Shetter JD, 1981: Sources of atmospheric methane: Measurements in rice paddies and a discussion. Journal of Geophysical Research, 86, 7203-7209. doi: 10.1029/JC086iC08p07203

Constantine W and Hesterberg T, 2021: splus2R: Supplemental S-PLUS functionality in R. R package version 1.3-3. https://CRAN.R-project.org/package=splus2R

Frenzel P, Rothfuss F and Conrad R, 1992: Oxygen profiles and methane turnover in a flooded rice microcosm. Biology and Fertility of Soils, 14, 84-89. doi: 10.1007/BF00336255

Gogo S, Guimbaud C, Laggoun-Défarge F et al., 2011: In situ quantification of $\mathrm{CH}_{4}$ bubbling events from a peat soil using a new infrared laser spectrometer. Journal of Soils and Sediments, 11, 545-551. doi: $10.1007 /$ s11368-011-0338-3

Holzapfel-Pschorn A, Conrad R and Seiler W, 1986: Effects of vegetation on the emission of methane from submerged paddy soil. Plant and Soil, 92, 223-233. doi: 10.1007/BF02372636

Komiya S, Noborio K, Katano K et al., 2015: Contribution of ebullition to methane and carbon dioxide emission from water between plant rows in a tropical rice paddy field. International Scholarly Research Notices, 2015, 8. doi: 10.1155/2015/623901 
Komiya S, Yazaki T, Kondo F et al., 2020: Stable carbon isotope studies of $\mathrm{CH}_{4}$ dynamics via water and plant pathways in a tropical Thai paddy: Insights into diel $\mathrm{CH}_{4}$ transportation. Journal of Geophysical Research: Biogeosciences, 125, e2019JG005112. doi: 10.1029/2019JG005112

Minamikawa K, Yagi K, Tokida T et al., 2012: Appropriate frequency and time of day to measure methane emissions from an irrigated rice paddy in Japan using the manual closed chamber method. Greenhouse Gas Measurement and Management, 2, 118-128. doi: 10.1080/20430779.2012.729988

Minamikawa K, Tokida T, Sudo S et al., 2015: Guidelines for measuring $\mathrm{CH}_{4}$ and $\mathrm{N}_{2} \mathrm{O}$ emissions from rice paddies by a manually operated closed chamber method. National Institute for Agro-Environmental Sciences, Tsukuba, Japan, pp. 76.

Myhre G, Shindell D, Bréon F-M et al., 2013: Anthropogenic and natural radiative forcing. In: Climate change 2013: The physical science basis. Contribution of working group I to the fifth assessment report of the intergovernmental panel on climate change. ed. by Stocker, TF, Qin D, Plattner G-K et al. Cambridge University Press, Cambridge, United Kingdom and New York, NY, USA.

Nouchi I, Mariko S and Aoki K, 1990: Mechanism of methane transport from the rhizosphere to the atmosphere through rice plants. Plant Physiology, 94, 59-66. doi: 10.1104/pp.94.1.59

R Core Team, 2020: R: A language and environment for statistical computing. R Foundation for Statistical Computing, Vienna, Austria. URL: https://www.R-project.org/

Schütz H, Seiler W and Conrad R, 1989: Processes involved in formation and emission of methane in rice paddies. Biogeochemistry, 7, 33-53. doi: 10.1007/BF00000896

Tokida, T. 2021: Increasing measurement throughput of methane emission from rice paddies with a modified closed chamber method. Journal of Agricultural Meteorology, in press. doi:10.2480/agrmet.D20-00029 
Tokida T, Adachi M, Cheng W et al., 2011: Methane and soil $\mathrm{CO}_{2}$ production from current-season photosynthates in a rice paddy exposed to elevated $\mathrm{CO}_{2}$ concentration and soil temperature. Global Change Biology, 17, 3327-3337. doi: 10.1111/j.1365-2486.2011.02475.x

Tokida T, Cheng W, Adachi M et al., 2013: The contribution of entrapped gas bubbles to the soil methane pool and their role in methane emission from rice paddy soil in free-air $\left[\mathrm{CO}_{2}\right]$ enrichment and soil warming experiments. Plant and Soil, 364, 131-143. doi: 10.1007/s11104-012-1356-7

Tokida T, Miyazaki T and Mizoguchi M, 2009: Physical controls on ebullition losses of methane from peatlands. In: Carbon cycling in northern peatlands. ed. by Baird, AJ, Belyea LR, Comas X et al. American Geophysical Union, Washington, pp 219-228.

Tokida T, Nakajima Y, Hayashi K et al., 2014: Fully automated, high-throughput instrumentation for measuring the $\delta^{13} \mathrm{C}$ value of methane and application of the instrumentation to rice paddy samples. Rapid Communications in Mass Spectrometry, 28, 2315-2324. doi: 10.1002/rcm.7016

Uzaki M, Mizutani $\mathrm{H}$ and Wada $\mathrm{E}, 1991$ : Carbon isotope composition of $\mathrm{CH}_{4}$ from rice paddies in Japan. Biogeochemistry, 13, 159-175. doi: 10.1007/BF00002775

Wang B, Neue HU and Samonte HP, 1997: Role of rice in mediating methane emission. Plant and Soil, 189, 107-115. doi: 10.1023/a:1004219024281

Wassmann R, Neue HU, Alberto MCR et al., 1996: Fluxes and pools of methane in wetland rice soil with varying organic inputs. Environmental Monitoring and Assessment, 42, 163-173. doi:

10.1007/BF00394048

Watanabe A, Murase J, Katoh K et al., 1994: Methane production and its fate in paddy fields: V. Fate of methane remaining in paddy soil at harvesting stage. Soil Science and Plant Nutrition, 40, 221-230. doi: 


\section{Figures}

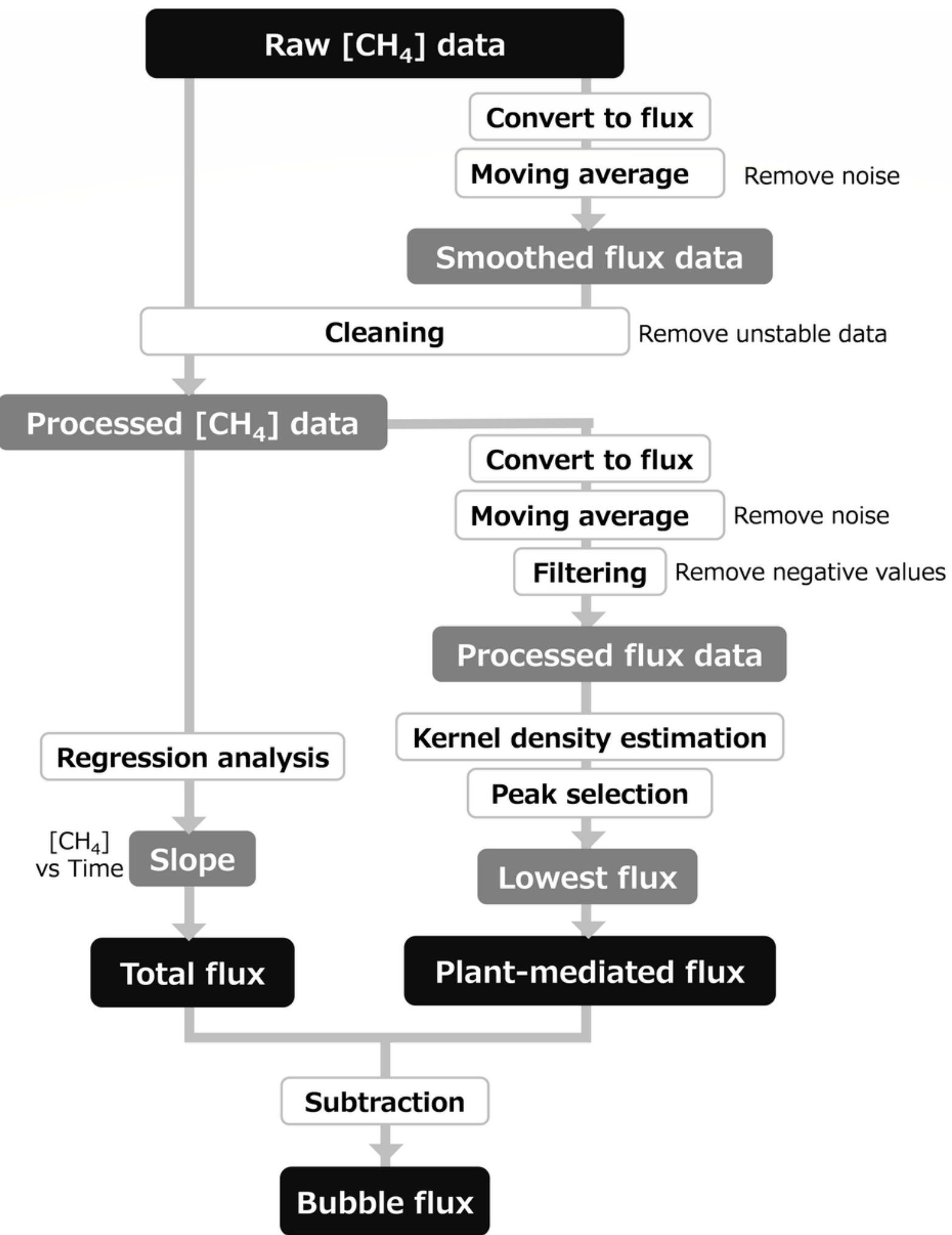

Figure 1 
Flow chart showing how $\mathrm{CH} 4$ emissions via rice plants and ebullition were separately estimated. Black boxes correspond to raw input and final outputs, gray boxes represent intermediate (partially processed) data, and white boxes indicate data processing steps.
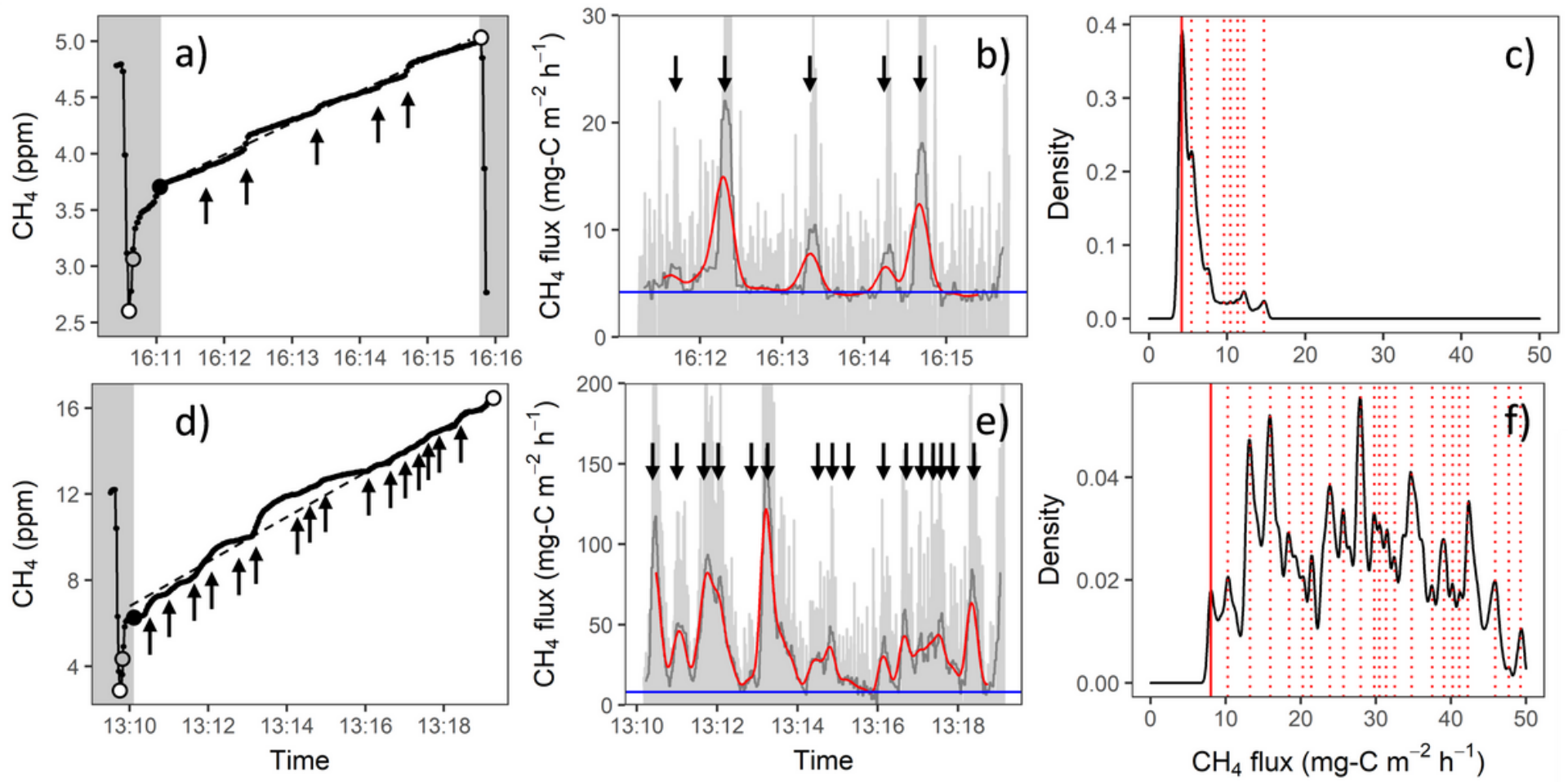

Figure 2

Data processing examples: $(a, d)$ time series of $[\mathrm{CH} 4]$ measured in the chamber; $(b, e)$ time series of the $\mathrm{CH} 4$ flux; $(c, f)$ the kernel density distribution of the fluxes. $(a-c)$ Methane flux measurements with few ebullition events (plot \#10, replicate 2, BT stage) and ( $d-f)$ ones with frequent bubbling events (plot \#6, replicate 2, HD stage). In (a) and (d), data in the shaded bands, which correspond to non-deployment and unstable measurement periods, were not used in the subsequent data processing. The dashed lines represent the fitted linear regression lines used to calculate the total $\mathrm{CH} 4$ flux. In (b) and (e), light gray, red, and blue lines indicate the original flux, the smoothed flux (by applying a moving average five times), and the plant-mediated flux, respectively. Dark gray lines, which indicate a less-smoothed flux curve obtained by applying the moving average only once, can be useful for identifying bubbling emissions (black arrows). In (c) and (f), vertical solid and dotted red lines indicate local peaks in the kernel density distribution of the flux; the plant-mediated flux was identified as the peak with the lowest flux intensity (red solid line). 
5-point

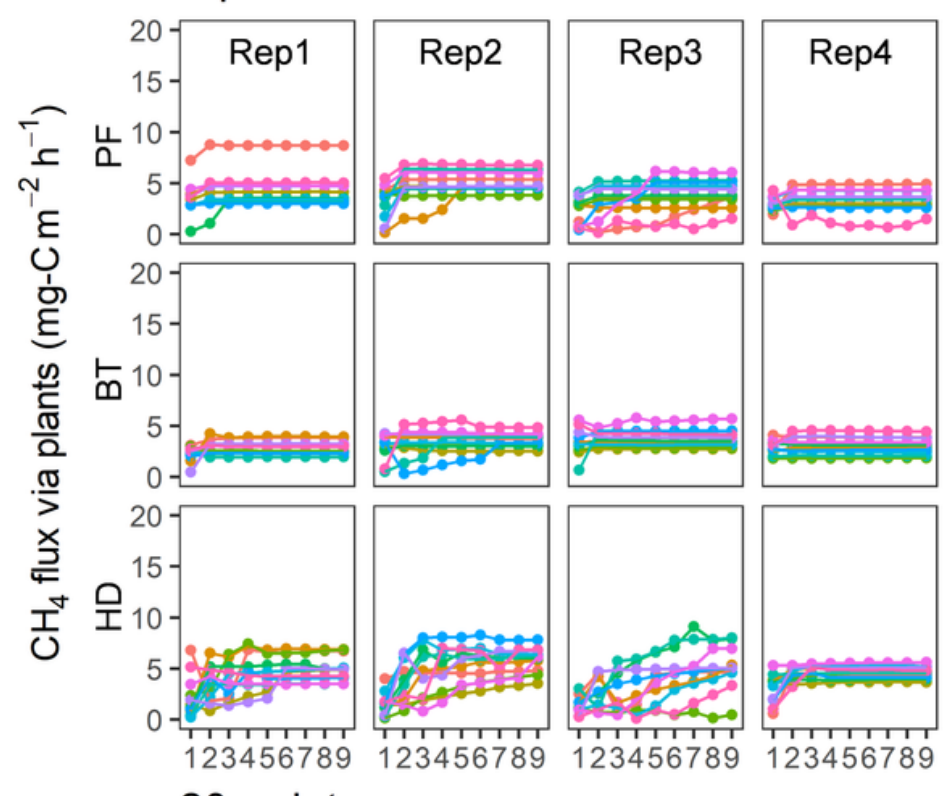

20-point

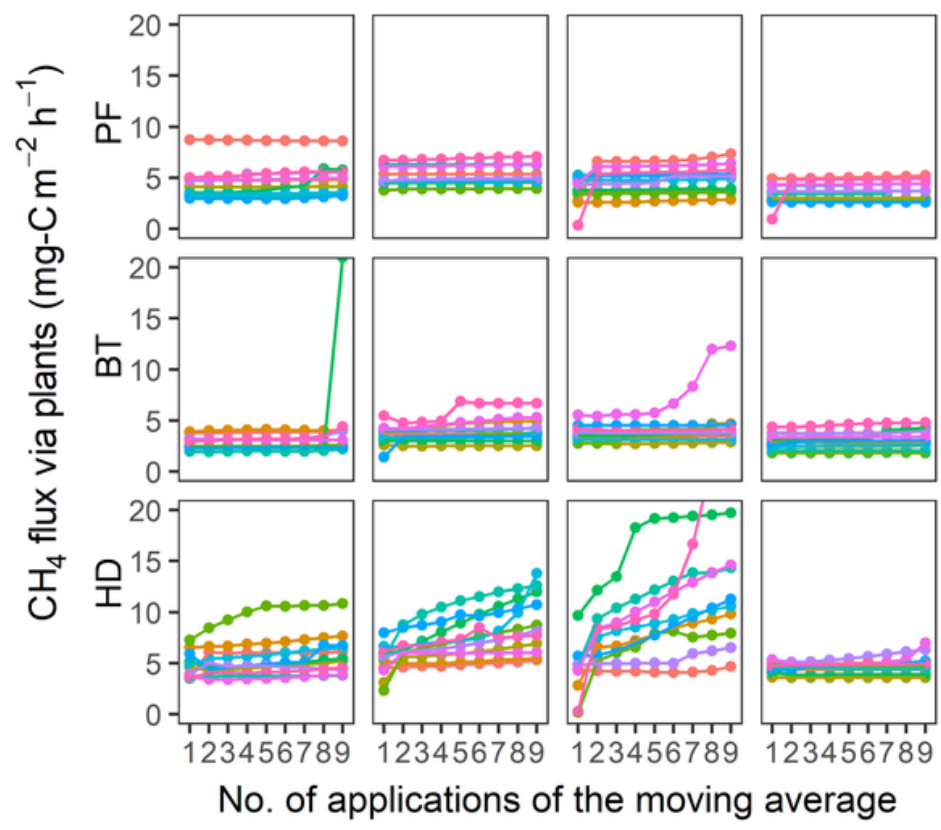

10-point

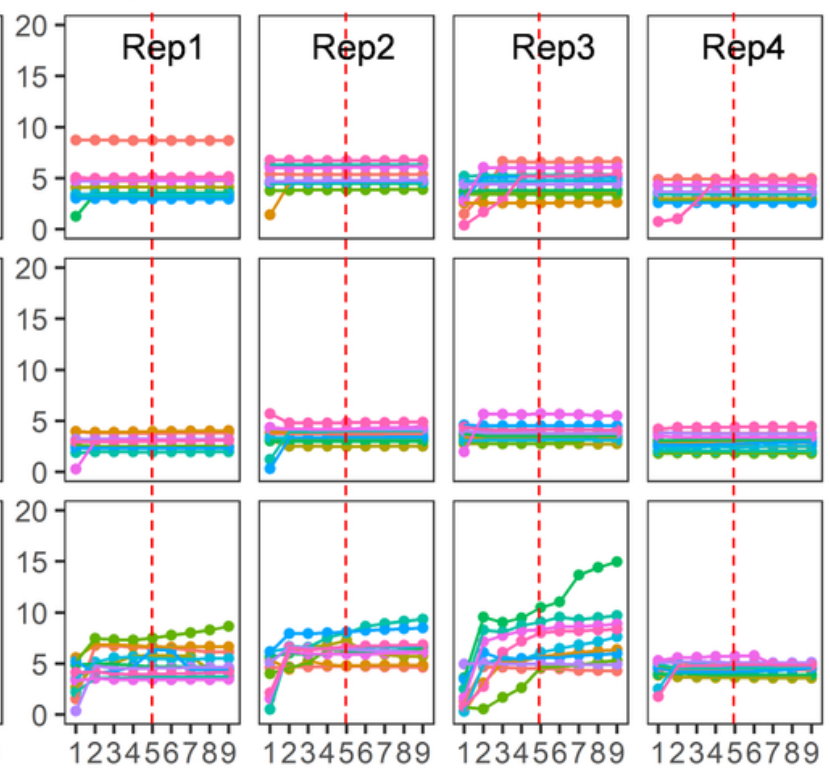

30-point
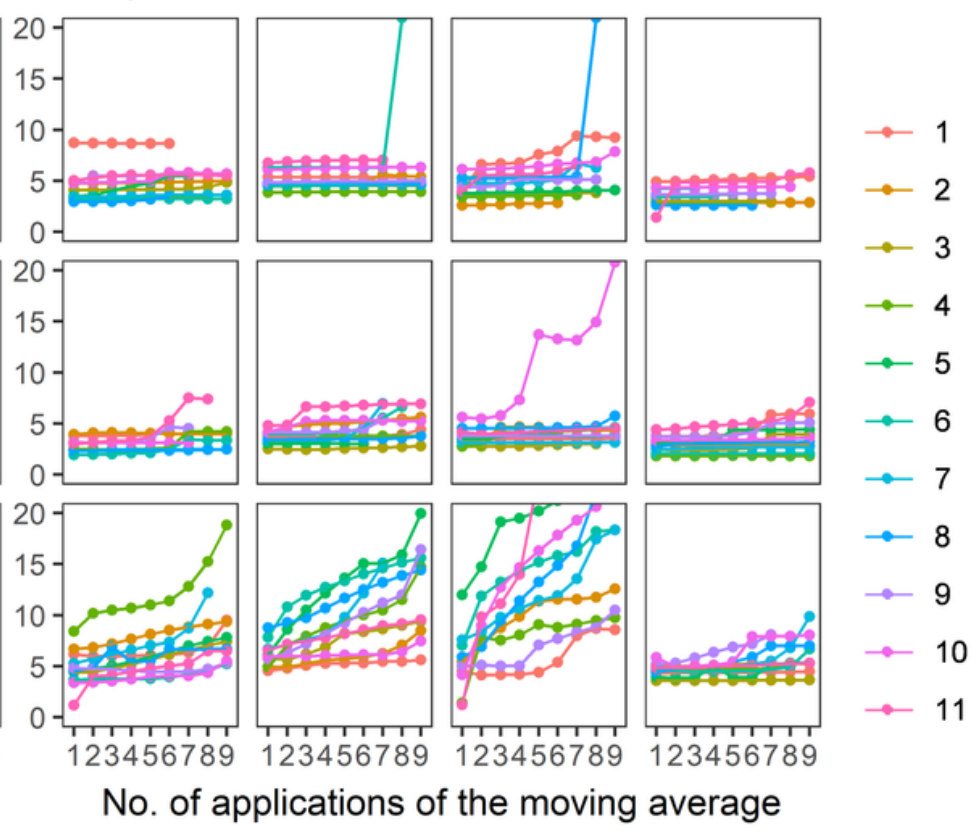

\section{Figure 3}

Plant-mediated fluxes estimated by using different moving-average protocols: averaging windows of 5 , 10,20 , or 30 data points and one to nine repeated applications of the moving average. Rep1 to Rep4 refer to the experimental replicates, each comprising 11 plots, indicated by the different colors, in the paddy field. The vertical red dashed lines indicate the protocol selected for use in this study (10-point moving average applied five times). 


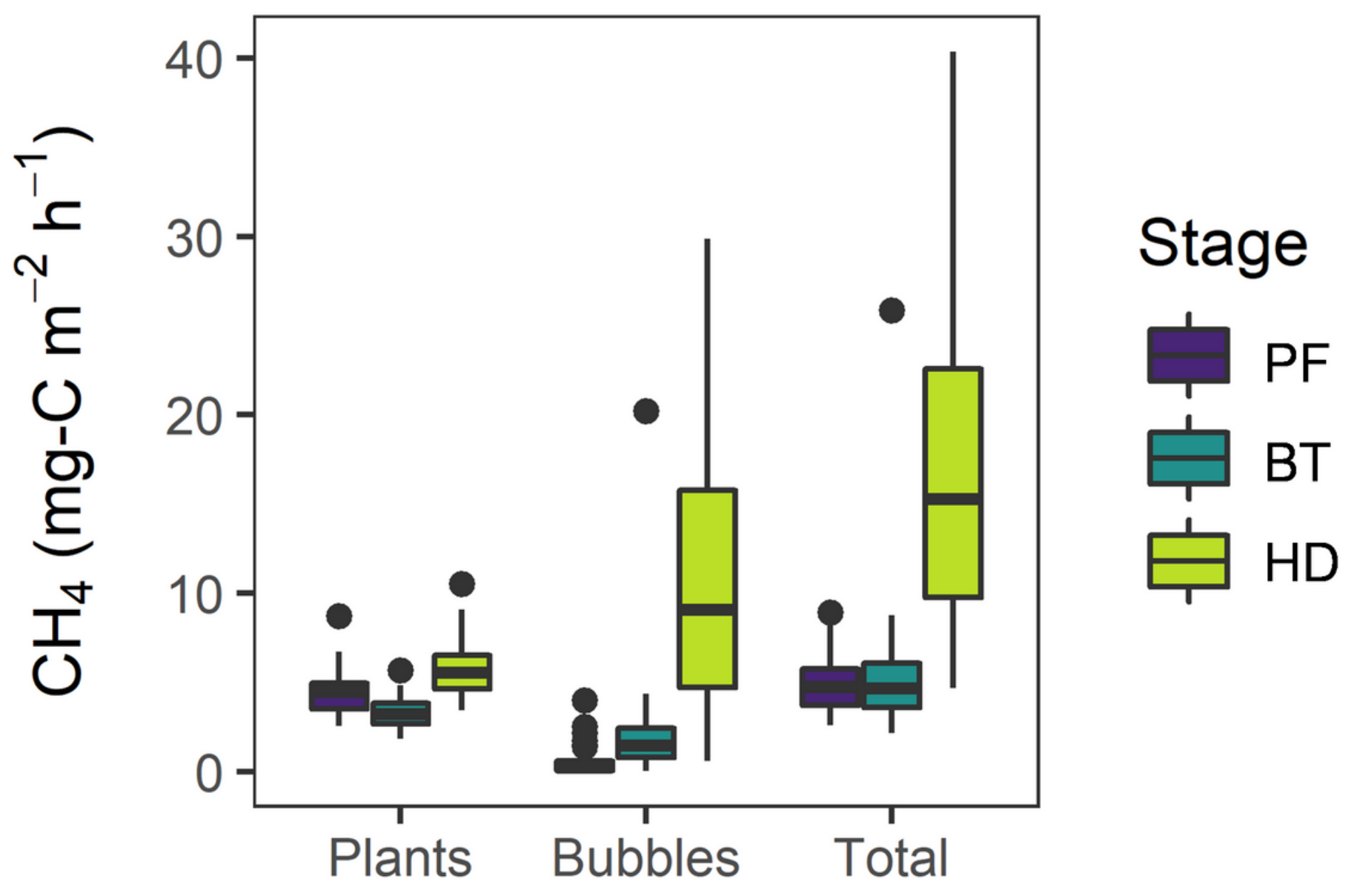

Figure 4

Boxplots of $\mathrm{CH} 4$ flux via rice plants and ebullition and of the total flux at different growth stages. PF, panicle formation; $\mathrm{BT}$, booting; and $\mathrm{HD}$, heading. The boxplots display a five-number summary: the minimum, the maximum, the sample median, and the first (upper) and third (lower) quartiles. Outliers (filled circles) were defined as data points that were located outside the whiskers of the box plot, namely, outside 1.5 times the interquartile range above the upper quartile and below the lower quartile. 


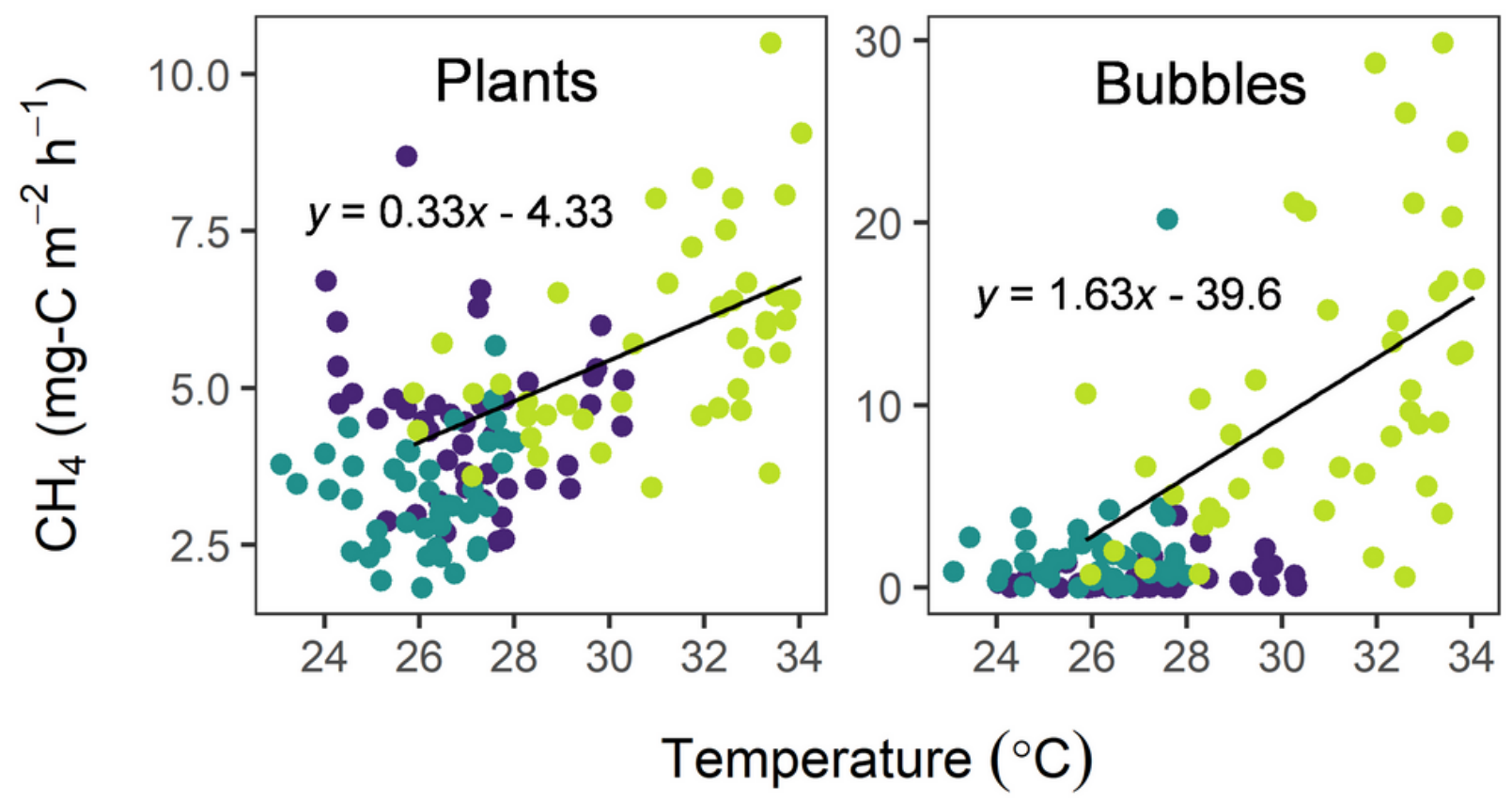

Stage

- PF

- BT

- $\mathrm{HD}$

Figure 5

Plant-mediated and bubbling fluxes as a function of air temperature. The black lines are the simple regression lines fitted to data obtained at the HD stage.

\section{Supplementary Files}

This is a list of supplementary files associated with this preprint. Click to download.

- msJAM2KajiuraRSSI.docx 